\title{
OPEN
}

\section{Author Correction: Surface plasmon enhanced Organic color image sensor with Ag nanoparticles coated with silicon oxynitride}

Sung Heo 10 , Jooho Lee, Gae Hwang Lee, Chul-Joon Heo, Seong Heon Kim, DongJin Yun, Jong-Bong Park, Kihong Kim, Yongsung Kim, Dongwook Lee, Gyeong-Su Park, Hoon Young Cho, Taeho Shin, Sung Young Yun, Sunghan Kim, Yong Wan Jin \& Kyung-Bae Park

Correction to: Scientific Reports https://doi.org/10.1038/s41598-019-57087-2, published online 14 January 2020

The original version of this Article contained a typographical error in the spelling of the author Jooho Lee, which was incorrectly given as Jooho lee.

In addition, the original version of this Article contained an error in Affiliation 6, which was incorrectly given as 'Department of Chemistry, Chonbuk National University, Jeonju, 54896, Republic of Korea'. The correct affiliation is listed below:

Department of Chemistry, Jeonbuk National University, Jeonju, 54896, Republic of Korea.

These errors have now been corrected in the PDF and HTML versions of the Article, and in the accompanying Supplementary Information file.

\begin{abstract}
(c) Open Access This article is licensed under a Creative Commons Attribution 4.0 International License, which permits use, sharing, adaptation, distribution and reproduction in any medium or format, as long as you give appropriate credit to the original author(s) and the source, provide a link to the Creative Commons license, and indicate if changes were made. The images or other third party material in this article are included in the article's Creative Commons license, unless indicated otherwise in a credit line to the material. If material is not included in the article's Creative Commons license and your intended use is not permitted by statutory regulation or exceeds the permitted use, you will need to obtain permission directly from the copyright holder. To view a copy of this license, visit http://creativecommons.org/licenses/by/4.0/.
\end{abstract}

(C) The Author(s) 2020 